\title{
Reseña histórica de la atención de los pacientes con enfermedades infecciosas en Santiago de Chile, hasta la creación del Hospital Dr. Lucio Córdova
}

\author{
ENRIQUE LAVAL R.
}

\author{
Brief description of the infectious disease patients in Santiago, Chile, \\ up to the creation of the Hospital Dr. Lucio Córdova
}

Sarampión toca la puerta viruela dice ¿quién es? y escarlatina contesta: "aquí estamos los tres".

(Cuarteta que se oía con frecuencia en Luna, en el siglo XVII)

En el año 1573, el rey Felipe II dictó la Ordenanza de Poblaciones, quizás la más antigua de la Colonia, destinada en parte a la ubicación de los hospitales. La ordenanza 122 que constituye la Ley $2^{\circ}$, libro IV, Título IV de la Recopilación de Indias establece textualmente:

"cuando se fundare o poblare alguna Ciudad, Villa o Lugar, se pongan los hospitales para pobres y enfermos de enfermedades que no sean contagiosas, junto a las Iglesias y por claustro de ellas y para los enfermos de enfermedades contagiosas en lugares levantados, y partes, que ningún viento dañoso pasando por los hospitales, vaya a herir en las poblaciones".

Sin embargo, debieron pasar casi trescientos años para que se planteara la necesidad de contar con hospitales o dependencias dentro de ellos para la atención exclusiva de pacientes con enfermedades infecciosas.

En 1871, siendo Presidente de la República don Federico Errázuriz Zañartu y a instancias de los Ministros del Interior y de Justicia. Culto e Instrucción Pública, señores Eulogio Altamirano y Abdón Cifuentes, respectivamente, debido a la escasez de camas en los dos hospitales que funcionaban en Santiago (San Juan de Dios para hombres y San Francisco de Borja para mujeres), se propuso el Gobierno construir un gran hospital en el barrio oriente de la ciudad gracias en parte al dinero donado por don Javier Casanova, consistente en cuarenta mil pesos y a erogaciones conseguidas en colecta del vecindario de la ciudad, que pronto superaron un cuarto de millón. Al conocer el éxito de la obtención de estos aportes privados el Gobierno decidió construir un segundo hospital en el sector norte de la ciudad.

El 7 de diciembre de 1871, el Presidente de la República decretó lo que sigue: considerando que el pensamiento de erigir un nuevo hospital al Oriente de Santiago, no se realizaría completa y felizmente, si no se funda a la vez un segundo hospital, más pe- queño que éste, destinado permanentemente para la curación de las enfermedades contagiosa, siendo la parte norte de la ciudad aquella en que con más ventaja puede establecerse.

El hospital de la zona oriente se denominó Hospital Del Salvador, destinado a atender "enfermedades comunes" para hombres, con capacidad de 500 camas y el de la zona norte Hospital de San Vicente de Paul, mixto, para enfermedades infecciosas agudas y con una cabida total de 400 camas. En todo caso, se resolvió dar preferencia al proyectado Hospital Del Salvador, colocándose la primera piedra el $1^{\circ}$ de enero de 1872. En marzo de ese año, se produjo una de las acostumbradas, pero "no por eso menos violenta", epidemia de viruela. El Intendente de Santiago, don Benjamín Vicuña Mackenna, designó una Junta de Lazaretos, que procedió a establecerlos en diferentes puntos de la ciudad, pero a instancias del Protomedicato y petición expresa del Gobierno se abrió el Lazareto Del Salvador el 24 de mayo de 1872, en las casas de la chacra adquirida a los Padres Mercedarios, en diciembre de 1871, llamada Chacra Barainca, en el barrio de Providencia y que en poder de aquellos se denominó de San Ramón Nonato. Estas casas fueron adaptadas para dar cabida a 100 camas. El rápido incremento de los enfermos obligó a aumentar su número, las que se establecieron en dos carpas del Ejército, con 30 camas cada una. En agosto la epidemia declinó y la Junta de Beneficencia de Santiago, acordó enviar al Lazareto Del Salvador, los enfermos convalecientes de los hospitales de San Juan de Dios y de San Francisco de Borja, para satisfacer la demanda de camas en esos establecimientos. Después, a fines de noviembre, ya prácticamente extinguida la enfermedad, se resolvió llevar los pocos variolosos existentes al Lazareto de la Maestranza (anexo al Hospital San Francisco de Borja). En 1876, a raíz de una epidemia de viruela, volvió a funcionar el Lazareto Del Salvador, utilizándose cinco salas construidas en 1873. Durante esta última epidemia se hicieron cargo del establecimiento las Hermanas de la Caridad.

En 1887 la .Junta de Beneficencia estimo que el Lazareto Del Salvador no debía continuar dedicado a la asistencia de variolosos, sino a la atención de "enfermos crónicos e incurables", para lo cual el 
gobierno concedió los fondos en abril de 1888 para continuar la construcción del hospital, que a partir del 31 de enero de 1889, pasó a llamarse oficialmente Hospital Del Salvador.

La Junta Directora de los hospitales Del Salvador y de San Vicente de Paul, comisionó el 9 de mayo de 1872 al arquitecto don Eugenio Chelli la confección de los planos del nuevo hospital de la zona norte de Santiago colocándose la primera piedra el $1^{\circ}$ de septiembre de dicho año. En esa oportunidad el miembro de la Junta de Beneficencia, don Enrique De Putron Cavareda pronunció un discurso en el cual dijo entre otras cosas: "que la junta encargada por el Presidente de Republica de los trabajos relativos a los nuevos hospitales, ha convocado a los miembros del Gobierno y al pueblo de Santiago para que venga a presenciar el acto solemne de la inauguración de los trabajos del Lazareto de San Vicente de Paul".

Sin embargo, los planos definitivos fueron ejecutados por el arquitecto don Carlos Stegmöller y en marzo de 1873 se encontraban casi terminadas las diez primeras salas. El hospital vino a abrir sus puertas solamente el 2 de noviembre de 1874 y a pesar de lo acordado anteriormente, fue diseñado para funcionar como hospital general.

En todo caso se construyo el "Departamento de San Roque" destinado a los enfermos de difteria, erisipela, lúes y enfermedades de la piel ocupando un patio independiente, colocado fuera del cuadro general de los edificios del hospital. Tenía cinco salas para enfermos, además de las piezas para diversos anexos. Este "Departamento" era exclusivo para hombres, pero además de las salas para las Clínicas de Medicina Interna, de Cirugía Ginecología, hubo otra para el aislamiento de enfermas con procesos infecciosos agudos.

Con motivo de la epidemia de viruela de 1876 el hospital fue evacuado, los enfermos llevados al Cuartel Cívico $\mathrm{N}^{\mathrm{o}} 2$, ubicado en la primera cuadra de la calle Santa Rosa y los variolosos ocuparon unos galpones que se construyeron en la parte posterior del hospital. Durante la Guerra del Pacífico, San Vicente de Paul quedo destinado exclusivamente para el Ejército y su sección Lazareto ocupada por variolosos y luego, por los prisioneros peruanos y bolivianos.

En 1890 desapareció el Departamento San Roque, instalándose una sala con el mismo nombre, para enfermedades infecciosas agudas.

La epidemia de viruela de 1893, obligó en abril a habilitar 140 camas en la sección mujeres, que aún se encontraba inconclusa.

En 1896, con el legado de \$10.000.- de don Joaquín Valledor, se edificó un nuevo departamento para enfermedades infecciosas agudas en especial para difteria, que había adquirido a comienzos de ese año gran desarrollo, circunstancia que obligó al Gobierno a designar una comisión médica, que en el hospital tomase a su cargo todo lo concerniente a la aplicación del suero antidiftérico. En 1900 en la sección de enfermedades infecciosas agudas se habilitó una nueva sala con 14 camas. Un año después se demostró que la letalidad por difteria era muy eleva- da, lo que se atribuyó a que el suero antidiftérico "se aplicaba fuera del tiempo".

Con el correr de los años, el Hospital San Vicente de Paul, fue transformándose en un establecimiento al cual se trasladaron la mayoría de las clínicas docentes, que funcionaban en los hospitales de San Juan de Dios y San Francisco de Borja. A principio de 1930 se hizo entrega del hospital al Ministerio de Educación y en junio de 1936, quedó definitivamente aprobado el plano del nuevo Hospital San Vicente de Paul, cuya construcción demoraría varios años, pasando a ser el Hospital Clínico José Joaquín Aguirre de la Universidad de Chile. ${ }^{3}$

El propósito del Gobierno de dedicar exclusivamente el Hospital San Vicente de Paul para las enfermedades infecciosas agudas no se cumplió, y por otra parte el Hospital Del Salvador que estaba destinado a ser un hospital general, debió convertirse transitoriamente en lazareto.

Las sucesivas epidemias de viruela y tifus exantemático que diezmaban la población de Santiago, obligaban de modo continuo a establecer hospitales y lazaretos provisorios que demandaban grandes gastos y como pareciera que la ciudad jamás se vería libre de ellas la Junta de Beneficencia en sesión del 28 de julio de 1886, designó una comisión integrada por don Miguel Fierro, don Miguel Dávila y don Eduardo Matte, para presentarle un proyecto de construcción de un lazareto definitivo. Esta comisión se constituyó en el Ministerio del Interior, presidida por el Ministro señor Zañartu y además de los miembros ya nombrados la integraron el Protomédico don José Joaquín Aguirre y los doctores Adolfo Valderrama y Vicente Izquierdo Sanfuentes, acordándose "ubicarlo el Noroeste de la ciudad en la ribera norte o Sur del río Mapocho, dotarlo con 500 camas (200 para hombre, 200 para mujeres y 100 para niños); en cada departamento colocar una sala de observación. Cada sala deberá contener 20 camas y sus dimensiones 30 metros de largo, 8 de ancho y 6 de alto, separada de la vecina por 15 metros. En los extremos oriente y poniente de las salas, se ubicarán cuatro piezas pequeñas para baños, excusados, guarda de útiles y cuidador. Finalmente, los servicios administrativos ocuparan el centro del edificio".

En septiembre de 1886, la Junta de Beneficencia quiso adquirir un terreno de propiedad de las Religiosas Agustinas, en Las Higueras de Zapata, en la banda norte del Río Mapocho frente a la calle Libertad, para construir allí el nuevo lazareto. Sin embargo, posteriormente la Junta se desistió de ese propósito y el Gobierno decidió construirlo contiguo al Cementerio General. El 5 de enero de 1887 fue designado Administrador del futuro lazareto, el Pbdo. Don Juan Achurra Matte.

Las primeras salas se construyeron por el arquitecto señor Ennes, a comienzo de 1887, bajo la urgencia de hospitalizar enfermos de cólera, epidemia que había estallado en el país en diciembre de 1886, recibiéndose los primeros enfermos en abril de 1887. El establecimiento fue denominado Lazareto del Norte o del Cementerio.

En abril de 1888, el lazareto fue cerrado, por ha- 
berse extinguido el cólera, destinado a entrar en funciones sólo en caso de aparición de viruela, pero reservando una sala de enfermos de cólera, si es que reaparecía la epidemia. Así, durante el año 1888 se atendieron a 340 enfermos de cólera, falleciendo 138 $(40,6 \%) ; 218$ de sarampión, con una letalidad del $5,5 \%$. Además, se asistieron escasos enfermos de viruela, varicela, erisipela, etc. En 1889 el lazareto pasó a llamarse de San José.

Desde el $1^{\circ}$ de mayo al 21 de diciembre de 1889 , los escasos enfermos de viruela permitieron a la Junta de Beneficencia destinar 45 camas para la atención de enfermedades comunes, las que luego hubo que desocupar en previsión de una nueva epidemia de viruela, que se suponía que estallaría con motivo de la llegada al país del vapor Burgundia, que traía inmigrantes españoles desde Málaga y en los que había aparecido la enfermedad. Ese año se atendieron 99 enfermos de sarampión y 151 variolosos, agregándose algo más de 300 enfermos de Medicina General y Cirugía. El médico era en esa época, el doctor Don Luis Alberto Figueroa, titulado en 1886.

A fines de 1890, la Junta de Beneficencia pensó suprimir el lazareto y construirlo en el primitivo sitio de Las Higueras de Zapata, para lo cual solicitó su opinión al Consejo Superior de Higiene. Al evacuar la consulta el Consejo expresó, entre otros considerándolo: "lo poco apropiado del edificio, para la clase de servicios a que se les destina y su proximidad al Cementerio General, cuyas fétidas emanaciones son arrojadas por los vientos sobre el mencionado Lazareto, hace necesario construir uno nuevo más apropiado. El terreno que para este fin se piensa destinar, se estima adecuado, siempre que entre el Lazareto y el cementerio de coléricos que allí existe, medie a lo menos un espacio de 200 metros". A pesar de todo el lazareto continuó contiguo al Cementerio General.

En febrero de 1891, se nombró médico del Lazareto de San José, al doctor Don Ricardo Dávila Boza, a quién se le confió la sección de mujeres, quedando en la sección de hombres, el doctor Don Luis Alberto Figueroa.

El doctor Dávila Boza, fue uno de los primeros médicos que se dedicó en Chile al estudio clínico y epidemiológico de las enfermedades infecciosas agudas, destacado higienista e ilustre Director del Instituto de Higiene de Santiago. En 1900 junto con el doctor Don Alejandro Del Río, integró la comisión para estudiar en la República Argentina la peste bubónica y en los años 1903 y 1907, le correspondió combatir la misma enfermedad en Arica e Iquique. En la memoria de su servicio correspondiente a 1891 presentada a la Administración del Lazareto, con precisión y franqueza expresó: "debo manifestar ante todo, que cuando me hice cargo del Lazareto, en los primeros días de febrero, se encontraba el establecimiento en tal estado de incuria, abandono, desaseo e inmundicia, que la primera idea que me surgió de su visita fue la probablemente ni entre los canes, ni entre los hotentotes se vería indiferencia semejante por los padecimientos de seres humanos. Por no parecer exagerado renuncio a toda descripción.
Básteme sólo recordar que los colchones eran simples montones de paja medio ensacados, que probablemente no se habían removido en muchos meses y que no se cuidaba de mantener convenientemente repartida en toda la extensión del lecho, por lo cual la cama era una verdadero tormento para el enfermo, en lugar de procurarle alivio y descanso".

Esta triste situación tuvo pronto arreglo porque en el curso de 1891, las Hermanas de la Caridad pasaron a prestar sus servicios al Lazareto de San José.

A fines de 1892 se desencadenó una nueva epidemia de viruela, que ocupó los hospitales Del Salvador, San Francisco de Borja y el Lazareto de San José. El Gobierno Otorgó \$14.000 para agregar en este último un nuevo pabellón y construir también otra sala para niños. La gravedad de la epidemia de 1892 movió al Pbdo. Achurra a plantear ante la Junta de Beneficencia la necesidad de declarar obligatoria la vacuna. El doctor Don Adolfo Murillo en esa época, demostró que desde 1864 a 1892 habían fallecido de viruela en Chile, 67.276 personas.

En octubre de 1892 el Consejo Superior de Higiene acogió públicamente y con gran revuelo ante la opinión ciudadana, la denuncia de que en el Lazareto de San José se daban de alta variolosos que se encontraban en plena evolución. El hecho era inexacto y fue una ligereza del Consejo: "los que han tenido viruela de forma confluente -dijo el administrador- quedan con las manchas algo levantadas y aparecen a los ojos del público como que todavía tienen la enfermedad en el periodo de desecación, cuando en realidad están completamente sanos".

Por otra parte, el alta de los enfermos estaba sujeta a normas que se cumplían rigurosamente: "por poco que la viruela hubiera tenido tendencia a ser confluente, el enfermo antes de su egreso debía tomar tres baños reglamentarios, con distancia de dos, tres o más días, entre y otro; con dos baños sólo se permitía salir a los afectados de viruela discreta y con un baño, a los que no habían tenido viruela, si no sarampión, varicela, etc".

En esos años cada uno de los dos médicos, doctores Figueroa y Dávila, tenían 100 camas a su cargo, con una renta de sesenta pesos mensuales.

Las deficiencias anotadas por el doctor Ricardo Dávila a su ingreso en 1891, debieron ser motivo de atención preferente de la Administración, pues en su informe anual de 1893 dice: "lo que queda por hacer no es mucho, ni de grande urgencia, pero es conveniente no olvidarlo". (Se refería a la necesidad de dotar al Lazareto, con una estufa de desinfección para la ropa de los variolosos). Una medida curiosa adoptó la Junta de Beneficencia en el mes de junio de 1893: en vista de las frecuentes y a veces graves complicaciones oculares que muchas veces sufren los variolosos, acordó solicitar al Administrador del Lazareto, la colocación de cortinas azules o verdes en las salas para aminorar en lo posible los efectos de la luz.

Con motivo de la epidemia de difteria que se produjo en Santiago, en abril de 1896, se habilitó en el Lazareto de San José, las salas San Luis y Santa 
Filomena para la hospitalización de los pacientes. Entre mayo y octubre de ese año se atendieron 107 enfermos con difteria, falleciendo 33. Además se hospitalizaron 39 variolosos.

Desde 1893, el único médico era el doctor Dávila Boza, pero en 1896 se le designó como ayudante transitorio al estudiante de Medicina, don Francisco Navarro, quien en ese mismo año obtuvo su título profesional. En septiembre de 1896, el Ejército deseó hospitalizar a numerosos soldados que padecían de sarna, pero el Pbdo. Juan Achurra se opuso tenazmente, "a pretexto de que a los militares, era muy difícil de cuidar".

El año 1897 fue una fecha de especial significado en el funcionamiento del lazareto: cambió su nombre por el de Hospital San José y a indicación de don Juan Achurra Matte, se aceptó destinar 40 camas para la atención de hombres tuberculosos e igual número para mujeres. Desde entonces y por muchos años, el Hospital San José se dividió en dos secciones: una de variolosos y otra de tuberculosos. ${ }^{4,5}$

En la monografía intitulada "Chile en 1908" de Eduardo Poirier, Mariano Guerrero Bascuñán dice: "El Hospital San José es, en realidad un hospital de infecciosos. Aunque ni su ubicación, ni el área que ocupa, ni la disposición y distribución de sus salas llenan las condiciones de un hospital de esa clase".

Durante varias décadas el Hospital San José fue el centro de las especialidades de Tisiología y Tisiocirugía. En diciembre de 1914 el doctor Manuel Moreno practicó el primer neumotórax terapéutico o artificial. En 1929 gracias a transformaciones, reorganización y modernización dedicó exclusivamente su atención a la patología tuberculosa.

El Hospital San José es en la actualidad un hospital general, docente-asistencial, base de todas las acciones de salud del Servicio Metropolitano de Salud Norte de Santiago, inaugurándose su nuevo edificio en 1999..$^{7-10}$

En noviembre de 1915, los doctores Don Vicente Izquierdo, Ruperto Vergara, Roberto y Alejandro Del Río y los señores Don Germán Riesco, Abraham Ovalle y Salvador Izquierdo, propusieron a la Junta de Beneficencia de Santiago, la celebración de un Congreso de Administradores y Médicos de los hospitales del país. Fue esta reunión "el despertar de los médicos a lo largo de Chile, que actuaban sin visión de los problemas fundamentales de la organización de la Medicina y con ella se rubricó el propósito de dedicar esfuerzos sostenidos a mejorar los servicios hospitalarios y a prepararse técnicamente para participar en su dirección”. El último punto del temario de este Primer Congreso de la Beneficencia Pública, que se inauguró el 13 de septiembre de 1917, estuvo dedicado a "los hospitales especiales para infecciosos", insistiéndose una vez más en su importancia, dada la gran morbilidad y letalidad que en esa época aún tenían las enfermedades infecciosas.

Con anterioridad, en 1900, debido a graves epidemias de sarampión y coqueluche, la mortalidad en Santiago llegó bruscamente a cifras altísimas, correspondiendo casi totalmente a los niños bajo 10 años de edad. A raíz de esto, don Manuel Arriarán
Barros, durante aquel año fundó a sus expensas el primer hospital para niños del país, que funcionó provisoriamente en la Casa de Ejercicios de San José, ubicada en la Calle Moneda. Terminada la epidemia, obtuvo del Gobierno un edificio fiscal que había servido para la Escuela de Normalistas, situado en la avenida Matucana y lo instaló allí. Poco después de la muerte del doctor Roberto Del Río, el nuevo establecimiento pasó a llevar su nombre. Con posterioridad la Junta de Beneficencia de Santiago recibió la suma de $\$ 800.000$ pesos, en 1907 , por disposición testamentaria de don Manuel Arriarán, quien falleció en aquel año, de los cuales, la mitad estaba destinada a la construcción de un hospital de niños en el sector Sur de la ciudad. La Junta adquirió en 1910 la quinta llamada El Mirador, ubicada en la calle Santa Rosa, próxima a la avenida Matta y acordó darle al futuro establecimiento el nombre de Hospital Manuel Arriarán. Las primeras 100 camas se habilitaron en 1919, por su Administrador el doctor Alejandro Del Río. En 1922 y proyectado por el mismo doctor Del Río, se inauguró el Pabellón para Enfermedades infecciosas Agudas, anticipándose en su concepción al sistema que años después se implantaría, como una reciente adquisición técnica, en el Hospital Claude Bernard de Enfermedades Infecciosas de París: el denominado "sistema de acordeón", que permite en un mismo pabellón aislar enfermos con diversas afecciones transmisibles agudas, sin que entren en contacto directo unos pacientes con otros, dando margen para aumentar el número de camas para determinada enfermedad, conservando siempre las normas fundamentales de aislamiento del enfermo y del personal, "con brillantes resultados".

Como ya se ha señalado, la necesidad de contar para la atención hospitalaria de Santiago con un establecimiento especializado para el aislamiento y tratamiento de pacientes con enfermedades infecciosas agudas transmisibles, había sido considerada desde hacía muchos años por las autoridades de la Beneficencia y Asistencia Social.

Bajo las inspiraciones de los Directores Generales, doctores Alejandro Del Río y Sótero Del Río, se dictó la Ley $\mathrm{N}^{\circ}$ 5.564, promulgada por el Presidente Arturo Alessandri Palma y publicada en el Diario Oficial el día 16 de enero de 1935. En ella "se dispone que anualmente se consulte en la Ley de Presupuesto la suma de $\$ 4.042 .500$, a fin de servir un préstamo no superior a $\$ 53.900 .000$, que contratará la Junta Central de Beneficencia, destinado a la construcción, reparación y habilitación de obras hospitalarias", asignándose la cantidad de $\$ 2.500 .000$ para la "construcción de pabellones destinados a la hospitalización de brotes epidémicos en Santiago y casa de convalecientes". En esa época el dinero consultado era suficiente para dar satisfacción a esa necesidad, pero vinculado con posterioridad su financiamiento a un empréstito contratado con la Caja de Seguro Obligatorio, sufrió tales tropiezos que su cumplimiento sólo fue parcial, desgraciadamente no pudo favorecer la iniciación del hospital para infecciosos agudos. 
Por fortuna la viruela dejó de ser epidémica a partir de 1923 (la vacuna antivariólica fue obligatoria desde 1918), pero el tifus exantemático provocó brotes epidémicos de gravedad. Entre 1918 y 1931 causó más de 10.000 muertos. En 1933 enfermaron 15.377 personas y murieron 3.560. La epidemia terminó en 1939 y ocasionó un total de 45.116 enfermos, de los cuales fallecieron 9.315. En 1934 la Beneficencia tenía destinadas 542 camas para la atención de los enfermos de tifus exantemático en Santiago, de los cuales 270 correspondían al Hospital Barros Luco, establecimiento que prácticamente era evacuado cuando aparecían estos brotes epidémicos, concentrándose allí el mayor número de pacientes. En consideración a la curva seguida en el país por las continuas epidemias de tifus exantemático, el doctor Alejandro Del Río propuso que el préstamo del cual ya comentamos, se utilizara para edificar pabellones ligeros", que el plano del Hospital Ramón Barros Luco, realizado por el ingeniero alemán Ruppel, consultaba hacia la calle Santa Rosa. Si bien la Junta aceptó la idea, esto no se pudo realizar por falta de fondos. Mientras tanto, la epidemia ascendía en forma vertiginosa y fue necesario clausurar la policlínica del hospital, para instalar más camas de aislamiento para tíficos.

A fines de 1938 entró a formar parte de la Junta Central de Beneficencia, el profesor doctor Lucio Córdova Labarca, siendo miembro de ella hasta abril de 1942. Dicho organismo prestó su aprobación a la indicación que el doctor Córdova formuló para proceder a la construcción en Santiago, de un Pabellón Modelo para la hospitalización de los enfermos infecciosos, anexado al Hospital Ramón Barros Luco, "no sólo destinado a cuidar enfermos, sino también a estudiar científicamente los múltiples problemas individuales como colectivos, que comportan las enfermedades infecciosas". Se designó a tres médicos que tendrían labor "full-time" para organizar dicho Pabellón y que habían sobresalido en el Servicio de Medicina y Enfermedades Infecciosas del Hospital Ramón Barros Luco, a cargo del profesor Córdova, por su preparación en bacteriología e inmunología, por sus conocimientos de la patología y clínica de las enfermedades infecciosas, por su dedicación entusiasta a la especialidad, cualidades puestas de relieve en numerosos trabajos presentados en la Sociedad Médica de Santiago y su publicación en diversas revistas científicas, dirigiendo además tesis para optar al título de médico, aceptadas con notas máximas por la Facultad. Estos tres médicos fueron los doctores Abraham Horwitz Barek, Roque Kraljevic Orlandini y José Perroni Barilari.

Todas las exigencias que constituyen las características de un Pabellón de Infecciosos, estuvieron a cargo del Jefe del Departamento Médico de la Dirección General de Beneficencia y Asistencia Social, doctor Enrique Laval Manrique, quien preparó el esquema necesario para la confección de los planos. El señor Luis Zorzi, arquitecto ayudante del Departamento de Arquitectura de la Dirección General, asesorado por su jefe don Clodomiro Valdivia, llevó a la confección de los planos las ideas plantea- das tan acertadamente, que fueron consideradas como modelo en su género, elogiadas con entusiasmo por los diferentes médicos e higienistas extranjeros que visitaron la construcción del pabellón. Así, gracias a la perseverancia y esfuerzos del profesor Lucio Córdova, unido al interés mostrado por el Director General de Beneficencia y Asistencia Social de la época, doctor Javier Castro Oliveira y por el doctor Enrique Laval Manrique, Jefe del Departamento Médico, se puso la primera piedra en 1939, en el Gobierno del Presidente Pedro Aguirre Cerda, siendo Ministro de Salubridad el doctor Salvador Allende Gossens.

Como se había acordado que este pabellón quedara anexado al Hospital Ramón Barros Luco y en consecuencia los servicios generales (administración, cocina, lavandería, farmacia, etc.) serían comunes, sólo se programó definitivamente los servicios de recepción, observación y hospitalización de enfermos, con sus anexos correspondientes.

La construcción del establecimiento tuvo varias interrupciones, hasta que finalmente la junta de Beneficencia obtuvo los recursos necesarios para continuar las obras, no sin antes haberse efectuado numerosas gestiones ante el Gobierno y Autoridades Universitarias. El lapso entre la "colocación de la primera piedra" la finalización de la obra gruesa y terminación del edificio, fue de 11 años. En dicha etapa los doctores Horwitz, Kraljevic y Perroni, efectuaron en el curso de sus becas en el extranjero, estadas y visitas a los hospitales especializados. A su regreso colaboraron en la planificación de la distribución definitiva de los distintos servicios, aconsejando también la construcción de un auditorio para la docencia y reuniones científicas, siendo aceptadas y realizadas todas sus sugerencias.

También en esa época los doctores Víctor Grossi y Pedro Martínez, Jefe Sanitario Provincial de Valparaíso y Jefe del Departamento Epidemiológico de la Dirección General de sanidad, respectivamente, en sendas comunicaciones esgrimieron sus razones para la creación de hospitales de infecciosos. Señalando que "en los países americanos existe muy escasa preocupación en orden a construir dichos establecimientos, a pesar de la morbilidad alta de las enfermedades infecciosas". Invocan razones sanitarias, demográficas, científicas y educacionales para dicho objetivo haciendo hincapié de que los principios que rigen la organización de un hospital de Enfermedades Infecciosas deben considerar personal idóneo, construcción y técnica adecuada. Las camas existentes actualmente en la República y las que se dispongan en el futuro estarán siempre ocupadas, por cuanto "estas enfermedades con frecuencia en forma epidémica y por lo menos endémica". Señalan que dadas "nuestras posibilidades económicas deben estar anexos a los hospitales generales", agregando finalmente que "de acuerdo a la población y las necesidades epidemiológicas de algunas localidades, así como la conveniencia de la enseñanza médica, debe recomendarse la creación de aquellos en Santiago, Valparaíso y Concepción". Vale la pena señalar que "por petición del consejero 
de la Junta Central doctor Hugo Grove, en 1938, se decidió la construcción en Valparaíso, de un establecimiento similar al aprobado en Santiago", lo que nunca fue llevado a cabo. ${ }^{12,16,17}$

Entre junio de 1941 y diciembre de 1942, Chile sufrió un brote inusitado de enfermedad meningocóccica, que de acuerdo al número de habitantes de Santiago y Valparaíso, presentó las características de una epidemia. Hasta el 31 de diciembre de 1942, se notificaron 5.885 casos, falleciendo 928 $(15,8 \%)$. En el norte la zona más comprometida fue la provincia de Antofagasta, con muy pocos casos en el sur del país.

Una vez más se evacuó el Hospital Ramón Barros Luco, en sólo 24 horas, disponiéndose de 400 camas para niños mayores de 7 años y adultos. En esta epidemia se utilizó el tratamiento con sulfamidados: sulfapiridina, sulfanilamida, sulfatiazol y sulfadiazina. Horwitz y colaboradores, de acuerdo a los resultados obtenidos con el empleo de la sulfadiazina, en la mayor casuística publicada, llegaron a la conclusión de que a futuro sería el fármaco de elección, ya que comprobó su gran poder bacteriostático unido a una acción tóxica mínima. La experiencia que significó el gran número de enfermos de enfermedad meningocóccica atendidos, sirvió para que el doctor Horwitz realizara su tesis de Profesor Extraordinario de Enfermedades Infecciosas. También esta epidemia constituyó un nuevo impulso para la construcción del Pabellón de Enfermedades Infecciosas del Hospital Ramón Barros Luco la que se termino en 1948. Dos años más tarde, en agosto de 1950, comenzó la habilitación del establecimiento, a raíz una epidemia de viruela menor, iniciada en el sur del país y que se extendió hasta Coquimbo. ${ }^{18}$

Así, los convalecientes de viruela y los nuevos casos de fiebre tifoidea, ocuparían las camas del primer piso del nuevo edificio cuya habilitación y equipamiento estaban finalizados.

Los objetivos a cumplir se pueden resumir en cuatro rubros: asistencial, de acuerdo con las características de su especialidad: normativo, formulación de normas terapéuticas para cada enfermedad infecciosa, para uso en el país; preparación del personal de otros hospitales, mediante cursos de entrenamiento: investigación clínica, terapéutica etiológica y epidemiológica; docencia, enseñanza de la clínica de las enfermedades infecciosas dentro del programa de estudios para médicos y enfermera además de cursos de postgrado para médicos especialistas en Salud Pública.

Esta fue la labor encomiable asumida por el doctor Roque Kraljevic, a través de sus funcionarios como Jefe de Servicio y Profesor, secundado por un grupo médico y de enfermeras comprometidos con el que hacer de este nuevo servicio del Hospital Barros Luco, el que a través del tiempo fue provocando admiración y adquiriendo prestigio. Al cabo de 12 años de intensa y fructífera labor, tanto asistencial como docente en las Escuelas de Medicina y de Enfermería de las Universidades de Chile y Católica, iniciada en el año 1944 en el antiguo Servicio de Medicina y Enfermedades infecciosas del
Hospital Barros Luco, las actividades se habían más que duplicado, llegando el Pabellón a constituirse en un Centro de Referencia Nacional de patología infecciosa. Todo esto junto con los problemas presupuestarios del Hospital Ramón Barros Luco, que no permitían el desarrollo y la mantención adecuada del edificio ni la renovación de equipos e instrumentos, llevaron al doctor Kraljevic a tomar la decisión de "luchar con los mejores argumentos, para obtener la separación e independencia del pabellón en relación a la administración del Hospital Ramón Barros Luco y transformarlo en un establecimiento con dirección y presupuesto independientes".

Finalmente, el $1^{\text {o }}$ de enero de 1963 , por acuerdo de la Dirección General y del Consejo del Servicio Nacional de Salud, pasó a obtener la categoría de Establecimiento con el nombre de Hospital de Enfermedades Infecciosas Dr. Lucio Córdova, nombre solicitado por el Profesor Kraljevic en recuerdo de su maestro en el antiguo Servicio de Medicina y Enfermedades Infecciosas del Hospital Ramón Barros Luco, quien como ya se ha destacado fue uno de los grandes impulsores de su construcción.

El Profesor Kraljevic continuó su brillante labor como Director del Hospital, hasta su jubilación en octubre de $1969 .^{15}$

El doctor Mario Salcedo Sepúlveda, ex Director del Servicio de Salud Sur de Santiago y digno sucesor del profesor Kraljevic en la cátedra, expresó con motivo del cincuentenario del Hospital, entre otras cosas: "el Hospital de Enfermedades Infecciosas ha sido un establecimiento ordenado, uno diría hasta disciplinado, respetuoso de sus tradiciones y en muchos aspectos, conservador. Así, como en el pasado supo asumir la atención de miles de enfermos con patologías endemo-epidémicas tradicionales de preferencia en personas jóvenes y previamente sanas, ha debido adaptarse al cuidado de adultos mayores con enfermedades crónicas y pluripatologías sépticas, que entrañan peor pronóstico y una previsible letalidad mayor. Con las transformaciones necesarias en su entorno, el hospital que estaba oculto por las ramas de árboles añosos y en mal estado, surgió luminoso, mostrando una estructura orgullosa de su tradición cincuentenaria”.

\section{Bibliografía}

1.- Laval M E. Régimen Legal de los hospitales durante la Colonia. Asoc. Ch Asist Suc 1935: 29: 3-50.

2.- Laval M E. Los treinta primeros años del Hospital del Salvador de Santiago. An Chil Hist Med.1971: 13:13-65.

3.- Laval M E. Apuntes para la Historia del Hospital San Vicente de Paúl. An Chil Hist Med 1972-73: 14-15: 119-60.

4.- Laval M E. Noticias sobre los Médicos en Chile. Tomo II (C-D-E). Ed Historia Médica. Santiago de Chile. 1970; p 177.

5.- Laval M E. Hospital San José (inédito).

6.- Poirier E. Chile en 1908. Impta. Barcelona. Santiago de Chile. 1909: p 210-8.

7.- Laborde M. Un pedazo de Historia. El Mercurio de Santiago. 22/10/2000.

8.- Edwards Ma, Yercovichil. Santamaría. EJ. La Tuber- 
culosis y el Hospital San José de Santiago. Rev Méd Chile 1983; 111: 315-8.

9.- El Mercurio de Santiago. 20/01 / 1996.

10.- Crónica. Hospital San José. Viejas dependencias comienzan a ser Historia. Perfil Médico 1998; 38: 6-12.

11.- Laval M E. Don Alejandro del Río. Rev. Asist. Soc 1944: 13: 210-21.

12.- Martínez P. Algunas consideraciones sobre hospitalización racional de infecto-contagiosos en Chile. Rev. Asist Suc 1940; 9: 236-46.

13.- Horwitz B A. Infección Meningocóccica en Chile. Impta. Stanley. Santiago de Chile. 1942 P. IX-XIV.
14.- Laval M E. Hospital para infecto-contagiosos agudos en Santiago. Asoc. Ch Asist Soc 1940: 11 6: 3-30.

15.- Kraljevic O R. Recuerdos de un viejo infectólogo. Pfizer. Santiago de Chile. 1998: P 40-2.

16.- Laval M E. Apuntes para la Historia del Hospital Ramón Barros Luco (inédito).

17.- Grossi V. Hospitales de Infecciosos. Rev. Asist Suc 1940: 9: 230-4.

18.- Palomino W C, González R O. Recuerdos de la epidemia de meningitis meningocóccica $(1941$ - 1942\} en el Hospital Barros Luco de Santiago de Chile. Rev Chil Infect 1990; 7: 23, 1-4. 